\title{
Energy Density and Radiation Losses in Giant Radio Galaxies
}

\author{
Marek Jamrozy \& Jerzy Machalski \\ Astronomical Observatory of the Jagiellonian University, ul. Orla 171, \\ 30-244 Krakow, Poland
}

\begin{abstract}
The volumes, equipartition energy density, and radiation losses due to the synchrotron and inverse Compton interactions as a function of redshift and projected linear size of Giant radio galaxies are discussed. The new results are based on data from three samples: 1) Ishwara-Chandra \& Saikia (1999), 2) Schoenmakers (Ph.D. Thesis, 1999), and 3) Machalski, Jamrozy \& Zola (2001).
\end{abstract}

\section{Introduction}

Giant radio sources with linear sizes greater than $1 \mathrm{Mpc}$ form an extreme class of extragalactic radio sources. The majority of them are radio galaxies at $z<0.3 \sim 0.4$. They are of special interest for studying a number of astrophysical problems. One of them is under what circumstances some sources evolve to "giant" sizes. These Giants must be extremely old and/or located in a very underdense environment, and/or have a stronger central source of energy as compared to smaller radio sources. It is very important to compare various physical parameters of normal-size and giant sources. Here we present a contribution to the following problems: 1) how does the energy density in the lobes of FRII-type radio sources evolve with redshift, and how does it relate to the evolution of energy and pressure of the intergalactic medium (IGM), and 2) how do radiative losses differentiate the lobes of giant sources from normal ones.

\section{Results}

\section{Correlation between the Energy Density and Redshift}

Using the Spearman partial rank correlation coefficients between the equipartition energy density in lobes $u_{m e}$ and redshift and/or power $P_{1.4}$ for 49 Giants with $D>1 \mathrm{Mpc}$, we deduced that the apparent correlation $u_{m e}-z$ arises from two others: $u_{m e}-P$ and $P-z$. We also checked the correlation between $u_{m e}$ and size $D$. The relevant partial correlations for 32 Giants with $10^{24.2} \leq P_{1.4}\left[\mathrm{~W} \mathrm{~Hz}^{-1} \mathrm{sr}^{-1}\right]<10^{25.0}$ show that there is a significant correlation $u_{m e}-D$ which implies the same between the energy density and volume. Therefore, there is no evidence that the undetected dependence between the energy density and redshift does not exist; it can be much weaker than the detected $u_{m e}-P$ and $u_{m e}-D$ correlations. The apparent distribution of the energy density vs. redshift is shown in Fig. 1, left panel.

Correlation between the Pressure Ratio and the Lobe Size

A non-relativistic, diffuse and uniform IGM in thermal equilibrium has an electron pressure, $p_{\mathrm{IGM}}$, which should increase with redshift as $p_{\mathrm{IGM}}(z)=p_{o}(1+z)^{5}$ 

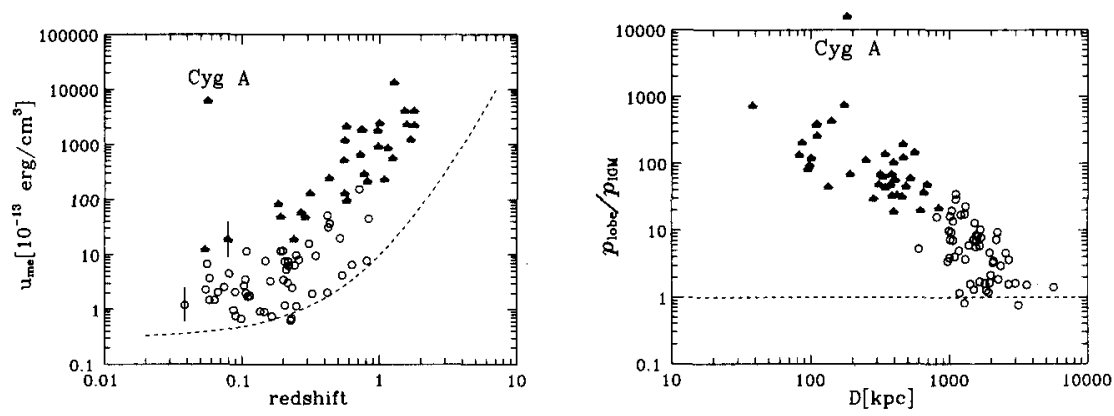

Figure 1. left panel: Apparent distribution of $u_{m e}-z$ in the sample of giants (open circles) and $3 \mathrm{C}$ sources (filled triangles) (cf. Alexander \& Leahy 1987, Leahy et al. 1989, and Liu et al. 1992) . Vertical bars mark the typical error of $u_{m e}$. The dashed line indicates the minimum energy density of the intergalactic medium (cf. Subrahmanyan \& Saripalli 1993). right panel: Distribution of $p_{\text {lobe }} / p_{\text {IGM }}$ vs. D. Symbols as in the left panel.

(Subrahmanyan \& Saripalli 1993). On the other hand, pressure in the lobes must be higher and can be determined from the equipartition energy density as $p_{\text {lobe }}=u_{m e} / 3$. We found that the correlation between $p_{\text {lobe }} / p_{\text {IGM }}$ and $D$ is very strong and significant. The newly discovered Giants confirm that the pressure in the lobes of these sources is close to that of the intergalactic (even intercluster) medium (see Fig. 1, right panel.

\section{Correlation between the Rate of Radiation Losses and Size}

Following Ishwara-Chandra \& Saikia, the dependence of the radiation losses due to synchrotron and inverse Compton interactions on the linear size of Giant and $3 \mathrm{C}$ sources has been analysed. The new data confirm that (i) there is a distinct upper limit for these losses which can be described by $B_{m e}^{2}+B_{\mathrm{IGM}}^{2} \propto D^{1.4}$ and (ii) the synchrotron losses diminish with increasing size of sources and tapers to a minimum value for Giants. This minimum is determined by the equivalent energy density of the microwave background radiation.

Acknowledgments. This contribution is supported by the State Committee for Scientific Research (KBN) under the contract 2PO3D 01517 and 2PO3D 00820 .

\section{References}

Alexander P., Leahy J.P., 1987, MNRAS 225, 1

Ishwara-Chandra C.H., Saikia D.J., 1999, MNRAS 309, 100

Leahy J.P., Muxlow T.W.B., Stephens P.W., 1989, MNRAS, 239, 401

Liu R., Pooley G., Riley J., 1992, MNRAS 257, 545

Machalski J., Jamrozy M., Zola S., 2001, A\&A, 371, 445

Schoenmakers A.P., 1999, Ph.D. Thesis, Univ. of Utrecht

Subrahmanyan R., Saripalli L., 1993, MNRAS 260, 908 